\title{
Image Enhancement based Improved Multi-scale Hessian Matrix for Coronary Angiography
}

\author{
Kaifeng Chen \\ Information Science and \\ Technology College \\ Dalian Maritime University \\ Dalian, 116026, China
}

\author{
Qingbo Yin \\ Information Science and \\ Technology College \\ Dalian Maritime University, \\ Dalian, 116026, China \\ Mingyu $\mathrm{Lu}^{*}$ \\ Information Science and Technology College \\ Dalian Maritime University \\ Dalian, 116026, China
}

\author{
Xiao Jia \\ Information Science and \\ Technology College \\ Dalian Maritime University \\ Dalian, 116026, China
}

\begin{abstract}
The coronary angiography image is easy to be affected by many factors, such as vascular thickness varied huge, complex background noise, uneven illumination intensity and so on. The coronary angiography image is more difficult to deal with compared with other similar medical images. By using Hessian matrix multi-scale vascular detection method, the vicinity of blood vessels will yield a lot of background noise, and the small tiny blood vessels become blurred or even lost, which seriously affect the experiment results. In this paper, an improved multi-scale Hessian matrix is presented, combined with morphological top-hat operation for the detection of coronary angiography. The experimental results demonstrate the effectiveness of the proposed method.
\end{abstract}

\section{Keywords}

Image enhancement; Hessian Matrix; Morphological top-hat; Vessel detection; Coronary angiography

\section{INTRODUCTION}

Coronary angiography is a commonly used and effective method to diagnosis of coronary atherosclerotic heart disease (CHD). It is relatively secure and reliable invasive diagnostic technology. It has been widely used in clinical and considered as the "gold standard" to diagnosis of coronary heart disease. Image enhancement technology is used to enhance the meaningful information for people or machines from a series of process images, and suppress unwanted information. The enhanced image will be more suitable to the specific application than the original image.

The analysis of blood vessels in coronary angiography image is of great importance in clinical applications. Medical experts can manually divide the vascular structure. However when it comes to huge numbers of images or images with large numbers of vessels, it is not only tedious, but also time consuming. The quantitative analysis of coronary angiography with the assistance of computer is useful on many occasions. It plays a significant role to improve the accuracy of diagnosis of heart diseases. But the contrast ratio of coronary angiography images is frequently low. What's more, it is susceptible to complex background or noise. To solve these problems, it's necessary to do the image enhancement for coronary angiography, so the subsequent processing of image segmentation and $3 \mathrm{D}$ reconstruction etc. will be more convenience in the future work.

To achieve an accurate vessel reconstruction, the process of vascular image enhancement for coronary angiography is used to suppress non-vascular structure and improve the profile of small tiny blood vessels. Many vascular image enhancement methods have been proposed, such as linear filters [1], morphology filter [2,3], anisotropic diffusion filter[7] and so on.

To enhance vascular image in a linear structure, the most common method is vascular enhancement filter based on Hessian matrix introduced in paper $[8,9,10,11,12,13]$. Multiscale fusion in the multi-scale vessel enhancement filter is used to solve the vascular characteristics that the size of blood vessel images is different. Appropriate scale range selection is the key to multi-scale linear filter, but the choice of the scale is a headache for people. To solve this problem, an improved Multi-scale Hessian matrix method is mentioned through combining Hessian matrix method with other image enhancement methods. Anisotropic diffusion technology is also proposed in the image enhancement for blood vessel angiographic images. However, this method is very sensitive to outliers, and it runs slowly because of its iterative nature. Rarely vessel enhancement methods use the method for the extraction of blood vessels. Compared with the image enhanced methods based on diffusion, top-hat enhancement method [3,4] can not only effectively suppress the local background, but also make the vascular structure become clearer. In this paper, a method of image de-noising and enhancement based on the improved multi-scale Hessian matrix that integrate the multi-scale Hessian matrix with morphological top-hat method for coronary angiography images is proposed.

\footnotetext{
* Corresponding author.

Email address: lumingyu@dlmu.edu.cn,

http://www.dlmu.edu.cn
} 


\section{MORPHOLOGICAL TOP-HAT OPERATIONS}

If the set $\mathrm{A}$ and $\mathrm{B}$ in the set $Z^{2}, A \ominus B$ means the corrosion of set $\mathrm{A}$ by set $\mathrm{B}$, which is defined as follows:

$$
A \ominus B=\left\{z \mid(B)_{z} \subseteq A\right\}
$$

If the set $\mathrm{A}$ and $\mathrm{B}$ in the set $Z^{2}, A \oplus B$ means the dilation of set $\mathrm{A}$ by set $\mathrm{B}$, which is defined as follows:

$$
A \oplus B=\left\{z \mid(\hat{B})_{z} \cap A \subseteq A\right\}
$$

The opening operation of set A by structural element B, the symbolic representation of which is $A \circ B$, is defined as follows:

$$
A \circ B=(A \ominus B) \oplus B
$$

Therefore, in mathematical morphology, The opening operation is the dilation of the erosion of set $\mathrm{A}$ by a structuring element $\mathrm{B}$. Morphological opening operation can be used to smooth the outline of objects, disconnect narrow neck, eliminate thin projections etc..

The highlights can be removed by the morphological opening operation, because the area of highlights smaller than the structure element. According to the truth, the structural element a little larger than the vessel diameter of the maximum size is chosen, and then do opening operation using the structural element obtained above will delete the entire vascular tree image of coronary angiography. Background image obtained by the opening operation is subtracted from the original image, the acquired vascular tree have got image enhancement, the process is known as top-hat operation. People frequently use the top-hat operation $[7,8]$ for image processing, the expression of which shows as follows :

$$
I=I_{0}-I_{0} \circ B
$$

Where $I_{0}$ is the original image, $B$ is a structural element, $I$ is the vascular image after image processing. Owing to the experiment results may be affected by different size and shape of the structure element, the chosen of the structure element is critical for top-hap operation. To enhance the vascular tree and make it clearer, the structural element selected is slightly larger than maximum diameter of the vessels. The disc-shaped structural elements are used in this paper.

\section{MULTI-SCALE HESSIAN MATRIX VESSEL EXTRACTION}

The Hessian matrix mentioned in paper [6] and [12] for a pixel at $(\mathrm{x}, \mathrm{y})$ is defined as:

$$
H_{\sigma}=\left[\begin{array}{cc}
I_{x x} & I_{x y} \\
I_{y x} & I_{y y}
\end{array}\right]
$$

Where $I_{x x}, I_{x y}, I_{y x}, I_{y y}$ refers to Gaussian second derivative for the image $I$.For multi-scale vessel enhancement filter, how to choose the appropriate range of spatial scales is the crucial factor, which seriously affected image enhancement effect of blood vessels. For simplicity and without loss of generality, the range of $\sigma$ is calculated with one-dimensional Gaussian filter. Supposing that one- dimensional signal is denoted by Gaussian function whose scale factor is $\sigma_{0}$ of the vessel in the paper [11]:

$$
y=A \exp \left(-x^{2} / 2 \sigma_{0}^{2}\right)
$$

Where A refers to the maximum brightness of blood vessels. For tubular structures, the response of the linear filter is the largest when the spatial scale factor $\sigma$ of Gaussian filter and vascular actual scale $\sigma_{0}$ matches best. The eigenvalues and eigenvectors of Hessian matrix represent the strength and direction properties of the vessels respectively. For the purpose of enhance all blood vessels in the range of scales, the convolution between the image and a Gaussian second-order derivative of the spatial scale factor $\sigma$ is got as follows:

$$
I_{m n}(\sigma)=I * \frac{\partial^{2} G(x, y ; \sigma)}{\partial m \partial n}
$$

Let $\lambda$ be eigenvalue of Hessian matrix, and supposing that $\left|\lambda_{1}\right| \leq\left|\lambda_{2}\right|$. The Blood vessel region can be discovered through the property of Hessian matrix's eigenvalue. The ideal vascular structure has the following characteristics: $\left|\lambda_{1}\right| \approx 0 ;\left|\lambda_{1}\right|<<\left|\lambda_{2}\right|$. The method of vascular measure proposed by Frangi etc. described in the literature [8] and [13] have been used in this work, the formula is as follows:

$$
V_{F}(\vec{\lambda})=\left\{\begin{array}{lr}
0 & \text { if } \lambda_{2}<0 \\
\exp \left(-\frac{R_{B}^{2}}{2 \beta^{2}}\right)\left(1-\exp \left(-\frac{S^{2}}{2 \gamma^{2}}\right)\right)
\end{array}\right.
$$

Where parameter $\beta$ and $\gamma$ are the weighting factors that measure the degree of their influence of $R_{B}$ and $S$ respectively. The value of $\gamma$ depends on the scales of gray image. $R_{B}=\lambda_{1} / \lambda_{2}$ separate tubular structure and other local structure area, and $S=\sqrt{\lambda_{1}^{2}+\lambda_{2}^{2}}$ separate vascular structure and the background area. Eigenvalue is calculated by the formula (8), and then take it into vascular similarity function so that the response $V_{F}(\vec{\lambda})$ is obtained.

If the local structure of the pixels is tubular structure (blood vessel), $R_{B}=0$ is got and it's simple to deduce $V_{F}(\vec{\lambda})$ is close to 1.If the pixels are noise, the eigenvalues of $\lambda_{1}$ and $\lambda_{2}$ will be very small, and it is not difficult to deduce $V_{F}(\vec{\lambda})$ is small. So the range of $V_{F}(\vec{\lambda})$ can be determined in the range of $(0,1)$. What's more, it's necessary to know that only when the local structure of the pixels is the tubular structure (blood vessel), the value of $V_{F}(\vec{\lambda})$ is the largest. The value of $V_{F}(\vec{\lambda})$ will be close to zero when it refers to other local structures or noise.

During the experiment, because of the vessels shown in the image contain different diameters, a method of multi-scale Hessian matrix is used, which makes it possible to describe the blood vessel image in different diameters. The Hessian 
matrix is calculated by second-order Gaussian derivative at multiple scales, and the largest response is selected in paper [9]:

$$
V=\max _{\sigma_{\min } \leq \sigma \leq \sigma_{\max }} V_{F}(\vec{\lambda})
$$

Fig.1 shows the image enhancing effect for coronary angiography where Fig.1-a is the original image, Fig.1-b is the multi-scale blood vessel image for Fig.1-a. It can be noticed that most of the background of the image is removed by a lower response of $V_{F}(\lambda)$. But due to the limitations of the Hessian matrix, resulting in a lot of background noise (aperture etc.), and many small tiny blood vessels disappeared at the same time. To be able to solve these problems, an improved method combining multi-scale Hessian matrix with morphological top-hat operation is proposed in this paper.

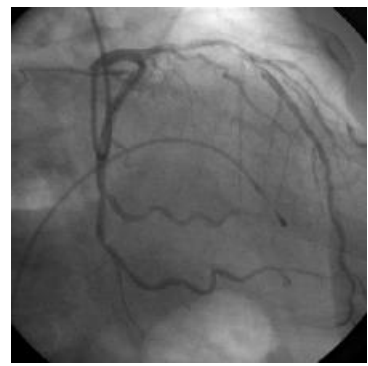

a

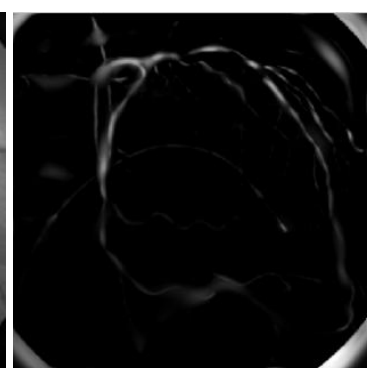

b
Fig. 1: Enhancing result by multi-scale Hessian matrix

a. The original image

b. Result by multi-scale Hessian matrix

\section{MULTI-SCALE HESSIAN MATRIX WITH TOP-HAT OPERATION}

In our work, the multi-scale Hessian matrix is used to desalinate the background noise combining with morphological top-hat method to solve the problems above. The flow chart for the proposed method is shown in Fig.2. Firstly it's necessary to gain the Hessian Matrix's eigenanalysis of each pixel of the image at different scales [10,11].

Vesselness value $V_{F}(\lambda)$ for each pixel of the image can be obtained easily. For convenience and easy to implement, different $V_{F}(\lambda)$ were achieved corresponding to different scales $\sigma\left(\sigma_{\min } \leq \sigma \leq \sigma_{\max }\right)$ after the image processing. The structure element is selected, then the morphological top-hat operation is executed to each $V_{F}(\lambda)$ corresponding to different scales $\sigma$ before the maximum value of $V_{F}(\lambda)$ is selected out. To improve the experimental results, making the final step of de-noising on $V$, i.e. execute the top-hat operation to $V$ who is the maximum value of $V_{F}(\lambda)$.

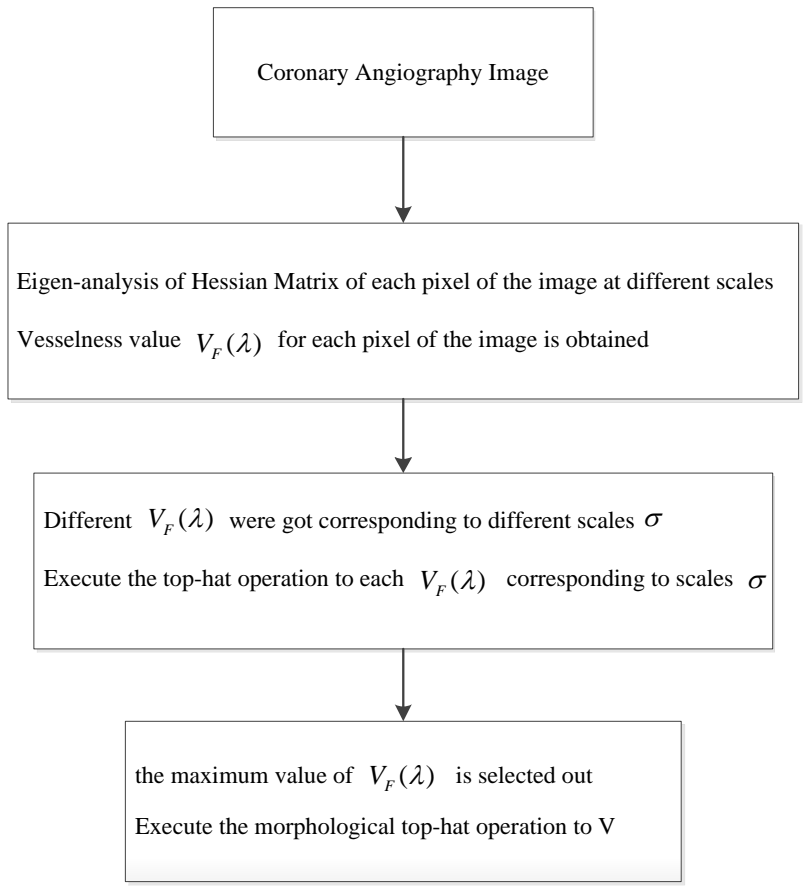

Fig 2: Flow chart for the proposed method

\section{EXPERIMENTAL RESULTS}

Fig. 3 depicts the enhancing results for coronary angiography image. Fig.3-a is the original image. Fig.3-b shows the enhancing result by the proposed method without threshold processing. Background noise almost completely disappeared, but some tiny blood vessel details cannot be reserved well. By observing the experiment data of the small tiny blood vessels, a threshold based on experience to bold small tiny vessels is set, and the results shown in Fig.3-c. By using the improved method most of the small tiny blood vessels get complete reserved, and the noise has been suppressed effectively at the same time. Compared with vessel skeleton extracted by another similar method of multi-scale Hessian matrix mentioned in paper [14] shown in Fig.3-d, obviously using the improved method proposed in this paper obtained better enhancement effect for the same coronary angiography picture.

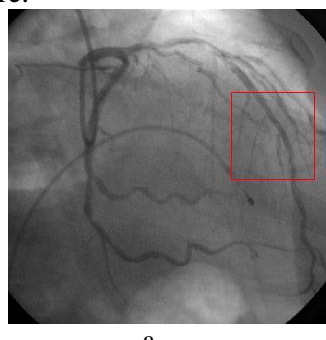

a

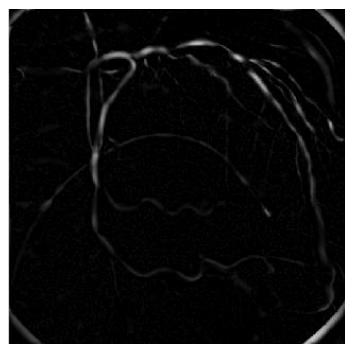

c

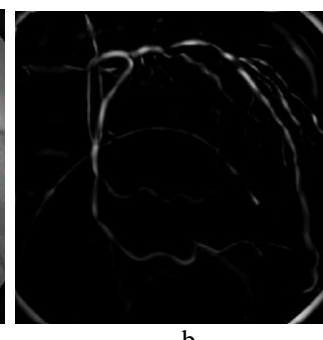

b

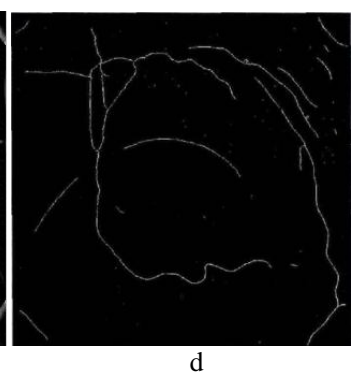

Fig. 2: Enhancing results for coronary angiography image 
a. The original image

b. Result by the proposed method without threshold processing

c. Result by the proposed method

d. Result by another method of multiple Hessian matrix

Fig.4 depicts the different image processing results for an enlarged view of the area in the red square of the original image Fig.3-a. Fig.4-a shows the local original image, and the image is blurred. Fig.4-b shows the result of processing by multi-scale Hessian matrix, it is not difficult to find that many edge details are lost. What's more, although it is not very obvious, you will find some halo surrounding the vessel if you look closely. Fig.4-c shows the proposed method in the paper. The thick blood vessels become thicker and the small tiny vessels preserve well simultaneously. Fig.4-d is the zoomed view of Fig.1-e. Obviously, the effect of Fig.4-d is not satisfactory compared with the proposed method.

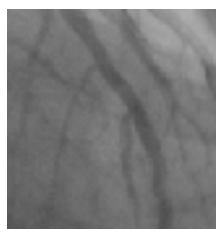

a

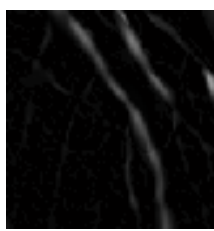

c

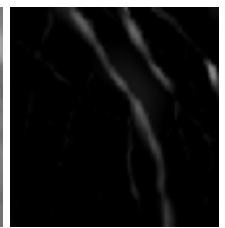

b

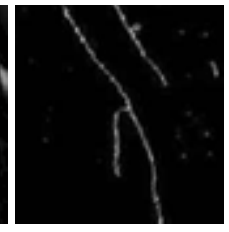

Fig. 3: Enlarged view of the area in the red square of the original image

a. The original image

b. Result by multi-scale Hessian matrix

c. Result by the proposed method

d. Result by another method of multiple Hessian matrix

\section{CONCLUSIONS}

An improved multi-scale Hessian matrix, combined with morphological top-hat operation for the detection of coronary angiography is presented in the paper. By using the novel proposed method in the process of image processing, people can effectively remove the background noise of images for coronary angiography while achieving the targets of image enhancement. The result of vascular extraction is overall ideal, and the small tiny blood vessels retained completely. The method proposed in the paper is simple and easy to implement. In the future work, the method refers to in this paper will be extended combining with other enhancement ways, and a new fast and effective image enhancement approach could be researched for coronary angiography.

\section{ACKNOWLEDGEMENTS}

This work was supported by The National Natural Science Foundation of China (NO. 61272369, No. 61175053 and No. 61370070) and Central Universities Fundamental Research Project (NO. 3132014325 and N0. 3132013335).

Firstly, I would like to show my deepest gratitude to my supervisor, Dr. Qingbo Yin, a respectable, responsible and resourceful scholar. Without his enlightening instruction, impressive kindness and patience, I could not have completed my thesis. Thank all of the teachers and students who offered me help in Intelligent Technology Researcher Center.

Last but not least, on the completion of this thesis, I am greatly indebted to my advisor Prof. Mingyu Lu who gave me valuable instructions and has improved me in language.

\section{REFERENCES}

[1] Poli, Riccardo, and Guido Valli. "An algorithm for realtime vessel enhancement and detection." Computer methods and programs in Biomedicine 52.1 (1997): 1-22.

[2] Sun, K. Q., and N. Sang. "Morphological enhancement of vascular angiogram with multiscale detected by Gabor filters." Electronics Letters 44.2 (2008): 86-87.

[3] Zeng, Ming, Jianxun Li, and Zhang Peng. "The design of top-hat morphological filter and application to infrared target detection." Infrared Physics \& Technology 48.1 (2006): 67-76.

[4] Bai, Xiangzhi, and Fugen Zhou. "Analysis of new tophat transformation and the application for infrared dim small target detection." Pattern Recognition 43.6 (2010): 2145-2156.

[5] Shi, Fei, and Jian Yang. "Multiscale vesselness based bilateral filter for blood vessel enhancement." Electronics letters 45.23 (2009): 1152-1154.

[6] Hooshyar, Sina, and Rasoul Khayati. "Retina vessel detection using fuzzy ant colony algorithm." Computer and Robot Vision (CRV), 2010 Canadian Conference on. IEEE, 2010.

[7] Manniesing, Rashindra, Max A. Viergever, and Wiro J. Niessen. "Vessel enhancing diffusion: A scale space representation of vessel structures." Medical image analysis 10.6 (2006): 815-825.

[8] Frangi, Alejandro F., et al. "Multiscale vessel enhancement filtering." Medical Image Computing and Computer-Assisted Interventation-MICCAI'98. Springer Berlin Heidelberg, 1998. 130-137.

[9] Lindeberg, Tony. "Edge detection and ridge detection with automatic scale selection." International Journal of Computer Vision 30.2 (1998): 117-156.

[10] Chakraborti, Tapabrata, et al. "A self-adaptive matched filter for retinal blood vessel detection." Machine Vision and Applications 26.1 (2014): 55-68.

[11] Hiriart-Urruty, Jean-Baptiste, Jean-Jacques Strodiot, and V. Hien Nguyen. "Generalized Hessian matrix and second-order optimality conditions for problems withC 1, 1 data." Applied mathematics and optimization 11.1 (1984): 43-56.

[12] Bofill, Josep Maria. "Updated Hessian matrix and the restricted step method for locating transition structures." Journal of Computational Chemistry 15.1 (1994): 1-11.

[13] Liping Chen. "Blood vessel image enhancement and level set segmentation algorithm based on Hessian matrix." [D]. Diss. Hunan University, 2012.

[14] Qin Zhang. "The extraction method research for blood vessel trees of coronary angiography images." MS thesis. Dalian Maritime University, 2012. 